\section{A Scanner-based Root Image Acquisition Technique for Measuring Roots on a Rhizotron Window}

\author{
Shufu Dong ${ }^{1}$, Denise Neilsen, Gerry H. Neilsen, and Michael Weis \\ Pacific Agri-Food Research Center, Agriculture and Agri-Food Canada, 4200 \\ Highway 97, Summerland, B.C. VOH 1Z0, Canada
}

Additional index words. Malus domestica, root investigation, digital, correlation, method
In this study, a scanner-based technique was developed that allowed the direct capture of still digital root images in conjunction with a simple and quick method for root measurements using a computer image analysis system. Measurements were made on apple trees grown in rhizoboxes. The advantages of this technique over digital minirhizotrons include its ability to: 1) examine more roots in less time; 2) monitor larger portions of each individual root; and 3) extract roots for physiological measurements.

\section{Materials and Methods}

Rhizobox and plant culture. Open-top rhizoboxes $(45 \times 45 \times 45 \mathrm{~cm}$ in dimension) were constructed from 2 -cm-thick plywood with one transparent side/wall of 3-mm-thick acrylic sheet (Acrylite ${ }^{\circledR}$ FF Acrylic Sheet, CYRO Industries, Rockaway, N.J.). The transparent side/wall was covered with a plywood door, which, when closed between measurements, prevented the entry of light into the box. A 1-year-old 'Gala'/M9 apple tree was planted in the center of each box containing a mixture of 1 peat moss : 1 perlite : 1 sandy loam soil (by volume). The boxes were placed on the floor of a greenhouse, and trees were grown under normal greenhouse conditions (natural daylight; 16 to $26^{\circ} \mathrm{C}$ temperatures) for $\approx 5$ months. Root growth on the transparent wall of the boxes was periodically measured manually and the digital root images were collected with an adapted scanner.

Adaptation of flatbed scanner and collection of digital root images. An off-the-shelf Agfa e25 flatbed scanner (Agfa, Toronto, Canada) was adapted by attaching a piece of foam board to one side of the scanbed to match the scanning surface to the window of the rhizobox, as shown in Fig. 1. A laptop computer (Acer TravelMate, Tribeca Technologies, New York) with a USB port was used to operate the scanner. Before root images were captured, the lid of the flatbed scanner was removed and the door covering the transparent wall of the rhizobox was opened. The scanner was roots from soils (Heeraman and Juma, 1993, Wiesler and Horst, 1994). These methods are destructive, time consuming, and labor intensive, and they cannot be used for time course studies. In addition, root loss is unavoidable, especially for fine roots during the root harvest and soil excavation/washing process. Transparent wall techniques for observation of plant roots have been developed to measure root growth dynamics in situ (Taylor et al., 1990). A variety of the techniques have been described in the literature (Freeman and Smart, 1976; Sanders and Brown, 1978; Upchurch and Ritchie, 1983; Waddington, 1971), and the one most widely used in recent research

\footnotetext{
Received for publication 8 Nov. 2002. Accepted for publication 25 Feb. 2003. This work was partially funded by Washington Tree Fruit Research Commission and MII program of Agriculture and Agri-Food Canada. Authors thank Eugene Hogue, Pat Bowen, and Peter Toivonen for the review of the manuscript. The paper is PARC Summerland Contribution No.2193.

${ }^{1}$ To whom reprint requests should be addressed. E-mail address: dongs@agr.gc.ca
}

is the minirhizotron technique. Minirhizotron observations are nondestructive, allowing reelongation, branching, and turn-over, as well as root distribution through the soil profile (Eissenstat and Yanai, 1997; Eissenstat et al., 2001; Johnson et al., 2001; Jose et al., 2001; Parker et al., 1991; Taylor et al., This technique usually involves installing transparent tube in the soil and using a miniature video or digital camera to record root images. Translating qualitative information from the recorded images to quantitative data is a tedious, time-consuming process (Ingram and Leers, 2001; Patena and Ingram, 2000; Vamerali et al., 1999).

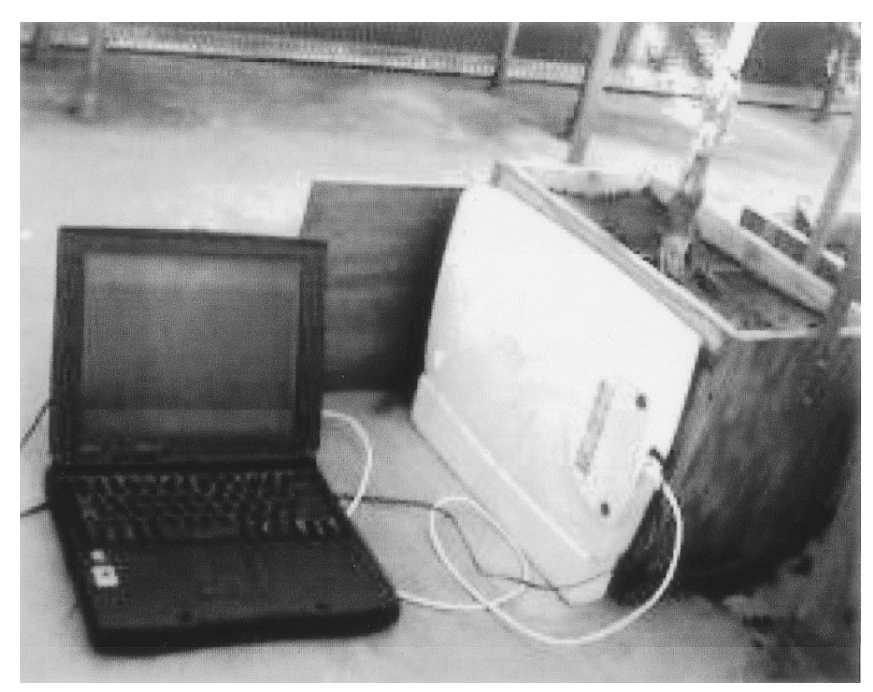

Fig. 1. General set-up of a computer-controlled scanner-based root image acquisition from a rhizobox. 
pressed against the transparent wall of the box by hand with the scanning side facing the wall. The images of roots visible through the transparent wall were periodically scanned at 150 dots per inch (dpi), saved to the laptop, and subsequently analyzed in the laboratory to determine root growth.

Measurement calibration. A computer image analysis system (CIAS; CID, Camas, Wash.) was used to measure root length of the collected images. Athree-point calibration was required before each set of root measurement. A calibration sheet (provided by CID), or a sheet with three points of known separation, was scanned under the same conditions as the captured root images. The special calibration procedure in the CIAS program was used to calibrate the measurement (CIAS User's Manual, CID). Once calibrated and stored, the calibration information was available to use for all subsequent measurements as long as the calibrated image was in the open file.

\section{Root length measurements}

Manual measurement. The length of roots growing on the transparent wall of the rhizobox was manually measured with a measuring tape only in the earlier stages of plant growth when just a few roots had appeared on the wall. At later stages there were too many visible roots to practically allow accurate root measurements with a measuring tape.

Computer tracing measurement. Using the trace measurement tool of CIAS, roots in the collected images were manually traced with the computer mouse. The length of the traced roots was automatically recorded after calibration.

Automatic measurement. A collected root image was first converted to gray scale using CIAS software, and the contrast of an image can often be improved by changing its intensity histogram (CIAS User's Manual, CID). The intensity histogram of a gray-scale image is a plot in which the number of pixels in the image is plotted as a function of the image's gray-scale level and, by adjusting the histogram stretch of the gray-scale image, the optimum contrast could be obtained that best distinguished roots from the background. The image was then remapped on a new image layer by defining threshold values of intensity. Using the CIAS automatic measurement tool, the total length of roots was automatically measured after calibration. Image layers are transparent planes existing over all images, which are used to mark an image without altering the actual image file (CIAS User's Manual, CID). The range of threshold intensity can be adjusted to give the best match of roots between the original image and the remapped layer. Black, brown, or white roots can also be distinguished from each other by careful adjustment of threshold intensity and separately measured.

Statistical analysis. The correlations between different measurements were calculated based on Pearson's correlation. All statistical analyses were performed with NCSS 97 Statistical System Software (NCSS Statistical Analysis Software, Kaysville, Utah).
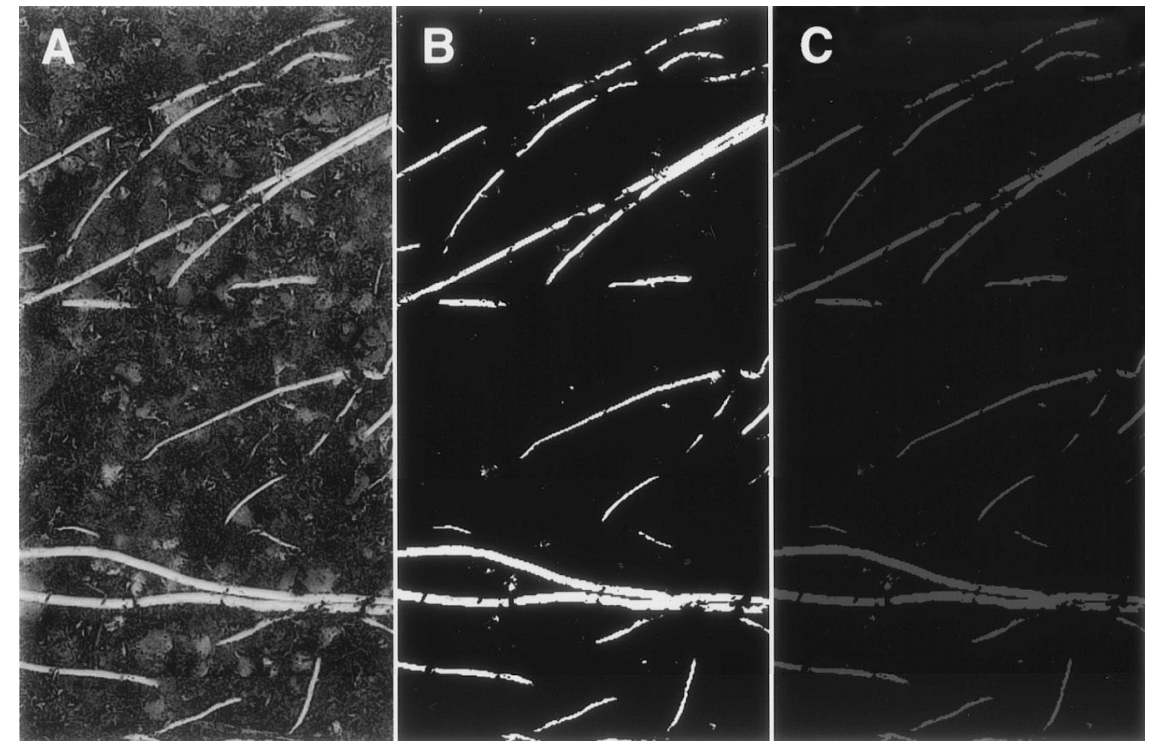

Fig. 2. (A) A part of original root image obtained with a regular scanner; (B) gray-scale image after conversion; and $(\mathbf{C})$ the remapped image for automatic measurement. The scale of the sampled image is $6.0 \times 3.5 \mathrm{~cm}$.

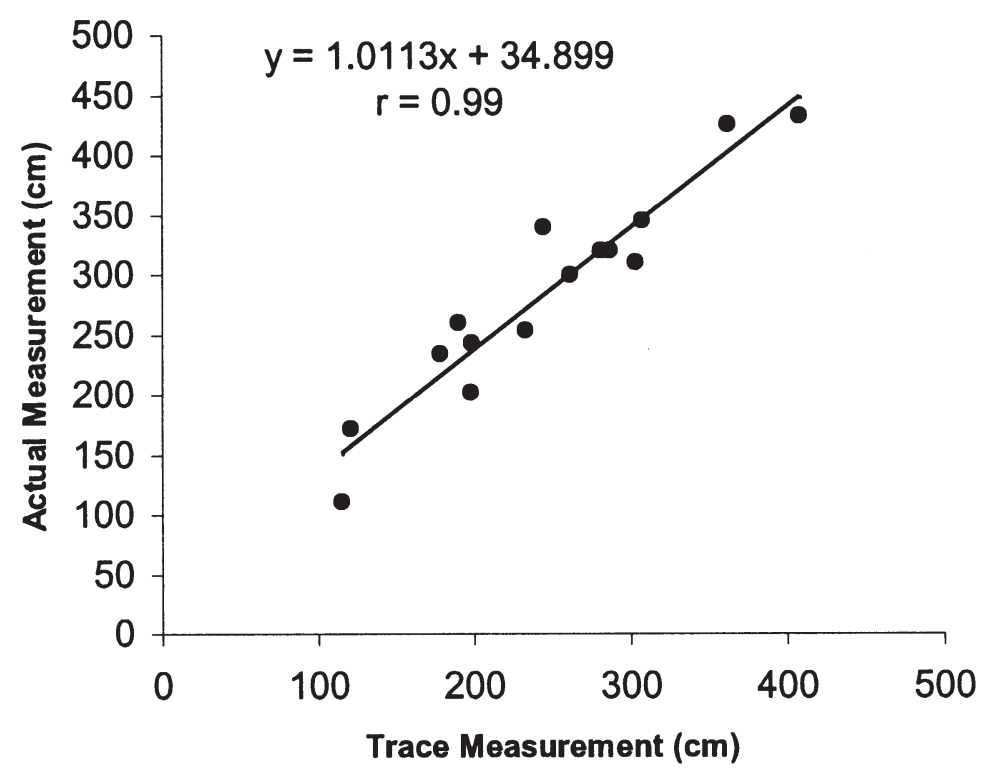

Fig. 3. Relationship between manual and computer tracing measurements of 'Gala'/M9 apple roots on the transparent wall of the rhizobox $(n=15)$.

\section{Results and Discussion}

Root image acquisition. High quality root images were obtained with an inexpensive flatbed scanner (Agfa e25) used in the rhizobox culture system (Fig. 2A). Auto brightness and contrast was turned off in order to standardize color intensity of images between rhizoboxes. The color image was used for the tracing measurement, but the image had to be converted to gray scale (Fig. 2B) and remapped on a new layer (Fig. 2C) before automatic measurement.

Relationships among manual, computer tracing, and automatic measurements. Root measurement data were pooled and correlations were made among different methods of measurement. Ahighly significant relationship was found between manual measurements and computer mouse-tracing measurements of roots visible on the transparent wall of the rhizobox $(P<0.0001)$ (Fig. 3). A significant linear relationship also existed between tracing and automatic measurements $(P=0.0010)$ (Fig. $4)$, and between actual and automatic measurements $(P=0.0013)$ (Fig. 5). The slope in Fig. 3 was almost equal to 1 (1.0113), which meant the manual and computer mouse tracing measurements were matched to each other, while slopes in Fig. 4 (0.6864) and Fig. 5 (0.7177) were considerably less than 1 , which suggested that the auto measurement overestimated root length, or the manual and computer mouse tracing measurements underestimated root 


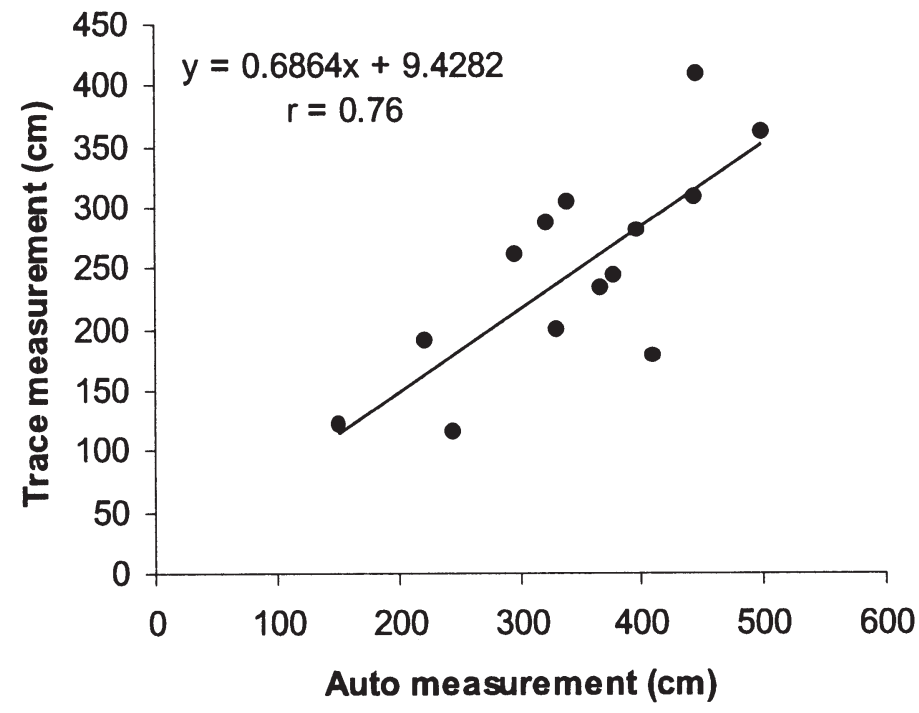

Fig. 4. Relationship between computer tracing and antomatic measurements of 'Gala'/M9 apple roots on the transparent wall of the rhizobox $(n=15)$.

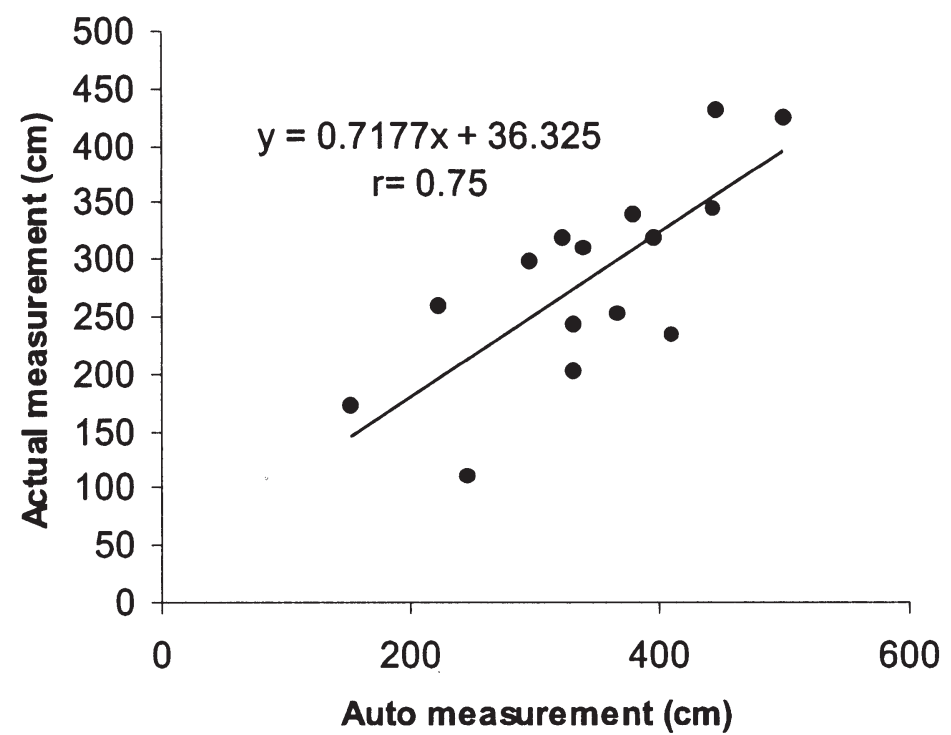

Fig. 5. Relationship between manual and automatic measurements of 'Gala'/M9 apple roots on the transparent wall of the rhizobox $(n=15)$.

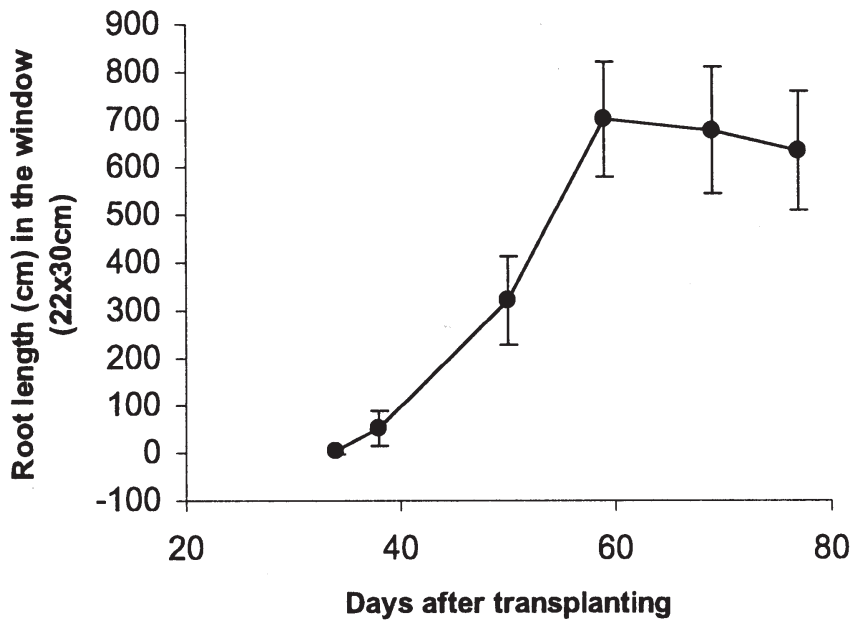

Fig. 6. Root growth dynamics of 'Gala'/M9 apple trees after transplanting in the rhizobox. Bars on data points represent standard errors of the mean of five replicates. Root length was automatically measured with the CIAS program. length. In this case, it is more possible for the auto measurement to overestimate the root length because there were perlite spots in the root medium, which interfered with the auto measurement.

Apple root growth dynamics after transplanting. Apple roots were first detected on the transparent wall of the rhizobox $34 \mathrm{~d}$ after transplanting. Thereafter, root growth gradually increased, reaching a peak at $59 \mathrm{~d}$ after transplanting, and then slowly declined (Fig. 6). At the growth peak, root intensity, defined as root length per unit area of the transparent wall, was $\approx 1.06 \mathrm{~cm} \cdot \mathrm{cm}^{-2}$.

A flatbed scanner was used in this study to directly capture digital images of roots at high resolution in a large area $(150 \mathrm{dpi} \times 22 \mathrm{~cm} \times 30$ $\mathrm{cm})$. The process took $\approx 1 \mathrm{~min} / \mathrm{image}$. With the help of CIAS software, the total root length in the image was automatically determined, and a significant relationship $(r=0.75)$ was obtained between manual and automatic measurements. Depending on the root numbers in the image, it took up to several hours to measure total root length of this large image manually, but only 2-3 min for automatic measurements regardless of the number of roots. Thus, this technique provides a simple and fast alternative tool to determine total root length of root images. There is also potential to measure different classes of roots (white, brown, and black), root surface area, and root diameter with the CIAS program. Although a few factors, such as soil physical properties, root age, and associated color, affect the accuracy of the automatic measurement in this study, the most significant is the uniformity of the soil medium, which can interfere with the identification of roots from the background (Heeraman et al., 1993; Vamerali et al., 1999). This background interference can be minimized by using image management techniques, but can never be eliminated because of soil heterogeneity. A uniform dark background soil will be the ideal medium to make CIAS assisted measurements, and white spots in soil profiles, which are similar to new roots in color, will result in inaccurate measurements. As the root number increases in the rhizotron window, roots may overlap each other, which will result in some errors in root measurement.

Although the scanning technique described in this paper was developed with rhizoboxes used in a greenhouse experiment, it can also be applied to on-site rhizotron/rhizobox techniques in field studies, and may possibly be extended to examination of soil profiles if a transparent window is installed in the soil.

\section{Literature Cited}

Eissenstat, D.M., L.J. Anderson, L.H. Comas, A Volder, and A. Lakso. 2001. Effects of irrigation and pruning on Concord grape root dynamics: Root physiology and longevity in relation to vine carbon status. HortScience 36:578-579.

Eissenstat, D.M. and R.D. Yanai. 1997. The ecology of root lifespan. Advances in Ecol. Res. 27:1-60.

Freeman, B.M. and B.E. Smart. 1976. A root observation laboratory for studies with grapevines. Amer. J. Enol. Viticult. 27:36-39.

Heeraman, D.A., P.H. Crown, and N.G. Juma. 1993. 
A color composite technique for detecting root dynamics of barley (Hordeum vulgare L.) from minirhizotron. Plant Soil 157:275-287.

Heeraman, D.A. and N.G. Juma. 1993. A comparison of minirhizotron, core and monolith methods for quantifying barley (Hordeum vulgare L.) and fababean (Vicia faba L.) root distribution. Plant Soil 148:29-41.

Ingram, K.T. and G.A. Leers. 2001. Software for measuring root characters from digital images. Agron. J. 93:918-922.

Johnson, M.G., D.T. Tingey, D.L. Phillips, and M.J. Storm. 2001. Advancing fine root research with minirhizotrons. Environ. Expt. Bot. 45:263-289.

Jose, S., A.R. Gillespie, J.R. Seifert, and P.E. Pope. 2001. Comparison of minirhizotron and soil core methods for quantifying root biomass in a temperate alley cropping system. Agrofor. Systems 52:161-168.

McCully, M.E. 1999. Roots in soil: Unearthing the complexities of roots and their rhizospheres. Annu. Rev. Plant Physiol. Mol. Biol. 50:
695-718.

Parker, C.J., M.K.V.Carr, N.J. Jarvis, B.O.Puplampu, and V.H. Lee. 1991. An evaluation of the minirhizotron technique for estimating root distribution in potatoes. J. Agr. Sci. 116:341-350.

Patena, G. and K.T. Ingram. 2000. Digital acquisition and measurement of peanut root minirhizotron images. Agron. J. 92:541-544.

Pepper,I.L. and D.E. Bezdcek. 1990. Root microbial interactions and rhizosphere nutrient dynamics. p. 375-410. In V.C. Baligar and R.R. Duncan (eds.). Crops as enhancers of nutrient use. Acad. Press.

Sanders, J.L. and D.A. Brown. 1978. A new fiber optic technique for measuring root growth of soybeans under field conditions. Agron. J. 66: $828-832$.

Schuurman, J.J. and M.A.J. Goedewaagen. 1971. Methods for the examination of root systems and roots. 2nd ed. Center for Agr. Publ. and Documentation, Wageningen, The Netherlands. p. 1-85.

Taylor, H.M., D.R. Upchurch, and B.L. McMichael.
1990. Applications and limits of rhizotrons and minirhizotrons for root studies. Plant Soil 129: 29-35.

Upchurch, D.R. and J.T. Ritchie. 1983. Root observations using a video recording system in minirhizotrons. Agron. J. 75:1009-1015.

Vamerali, T., A. Ganis, S. Bona, and G. Mosa. 1999. An approach to minirhizotron root image analysis. Plant Soil 217:183-193.

Volkmar, K.M. 1993. Acomparison of minirhizotron techniques for estimating root length density in soil of different bulk density. Plant Soil 157: 239-245.

Waddington, J. 1971. Observations of plant roots in situ. Can. J. Bot. 49:1850-1852.

Wells, C.E. and D.M. Eissenstat. 2001. Marked differences in survivorship among apple roots of different diameters. Ecology 82:882-892.

Wiesler, F. and W.J. Horst. 1994. Root growth of maize cultivars under field conditions as studied by the core and minirhizotron method and relationships to shoot growth. Z. Pflanzenernahr. Bodenk. 157:351-358. 